\title{
PATRÓN DE USO DE LOS MECANISMOS GRAMATICALES DE LA REFERENCIA POR ESTUDIANTES DE ESPAÑOL COMO SEGUNDA LENGUA
}

\author{
Pattern of use of grammatical mechanisms of reference by students \\ of Spanish as a second language
}

\section{Silvia Vargas Víquez*}

\begin{abstract}
RESUMEN
La comparación de datos que se presenta en este artículo se enmarca dentro de un estudio sobre la adquisición de los mecanismos de referencia en ESL en los tres niveles de proficiencia según ACTFL (2001). Esos recursos referenciales son: sustantivo común con determinante indefinido, sustantivo común con determinante definido, nombre propio, pronombre en tercera persona singular y sus determinantes posesivos y anáfora cero. Los resultados encontrados a partir de las muestras no nativas se contrastan con los obtenidos al analizar un corpus de hablantes nativos del español.
\end{abstract}

Palabras clave: adquisición, segundas lenguas, referencia, coherencia, cohesión.

\begin{abstract}
Data comparison presented in this article is demarcated within a research project on acquisition of "referent" mechanisms in SSL in the three levels of proficiency according to ACTFL (2001). Those referential forms are: a common noun with indefinite determinant, a proper noun, a third-person singular noun and their corresponding possessive determinants and an anaphora zero. The results from non-native samples are compared with those obtained by analyzing a corpus of native speakers of Spanish.
\end{abstract}

Key Words: acquisition, second languages, referents, coherence, cohesion.

\footnotetext{
*M.L. Silvia Vargas Víquez. Profesora Universidad de Costa Rica, Costa Rica.

Correo electrónico: silvarvi@yahoo.com

Recepción: 06-09-2017 Aceptación: 27-01-2018
} 


\section{Introducción}

La coherencia y la cohesión son propiedades del texto escrito reconocidas por el aporte que brindan a la construcción de sentido. La coherencia tiene que ver más con procesos mentales, acuerdos implícitos entre los hablantes y la unidad semántica; opera por medio de la cohesión textual a través de tres mecanismos principales: la puntuación, la referencia y la conexión (Calsamiglia y Tusón, 1999, pp. 221-229). Estos recursos cohesivos tienen la ventaja de manifestarse a través de elementos lingüísticos que se pueden contabilizar. La referencia, por su parte, repercute directamente en el significado, pues orienta la identificación de los diferentes participantes en el texto (Halliday y Hasan, 1976, p. 31). Tanto la coherencia como la cohesión y dentro de esta, la referencia- han servido de criterios de evaluación de una "buena escritura" y, por lo tanto, son de vital importancia en el aprendizaje de esta destreza.

Por esas razones, Alvarado-Gutiérrez y Vargas-Víquez (2009) realizaron una investigación que se concentró en el análisis de los mecanismos gramaticales de la referencia en sesenta redacciones escritas por estudiantes angloparlantes de español como segunda lengua (ESL), veinte por cada nivel de los propuestos por el American Council on the Teaching of Foreign Languages Inc. (ACTFL, 2001). En Vargas-Víquez (2012) se presentan los resultados del nivel principiante y en Vargas-Víquez (2014), los del nivel avanzado; en esta ocasión, se ofrecen los hallazgos de la comparación de los tres niveles de proficiencia (principiante, intermedio y avanzado) y, además, los que arrojó el análisis de un corpus de redacciones escritas por un grupo de hablantes nativos del español, todos ellos costarricenses adultos.

Las investigaciones que se encontraron sobre este tema en su mayoría contrastan la producción nativa con la no nativa. Un primer grupo contiene estudios que incluyen la cohesión 
como uno de los criterios para determinar la calidad de la escritura (o sea, si un texto está "bien escrito” o no), estos son: Shi (1993), Govardhan (1994), Robinson (1994), Vines (1997), Chiang (1999) y Pongsiriwet (2001). Un segundo grupo de investigaciones reúne las que analizaron los mecanismos cohesivos. Dentro de este último, por una parte, se hallaron dos trabajos sobre cohesión en general y que consideran la producción nativa como el modelo por alcanzar; en este sentido, Tornqvist (2004) coteja los mecanismos de coherencia en redacciones en inglés y español escritas por personas bilingües; mientras que Teijeira-Rodríguez, Van Esch y de Haan (2005) comparan la organización, coherencia y cohesión en argumentaciones de hablantes nativos del español con las de estudiantes holandeses que aprendían español como lengua extranjera. Y, por otra parte, se encontraron las investigaciones que se enfocan cada una en uno solo de los tipos cohesivos y que también comparan el uso nativo con el no nativo; primero, Reynolds (1996) trabajó la repetición, pero en el idioma inglés; segundo, Lee (2003) se concentra en recopilar datos empíricos sobre la adquisición de los pronombres clíticos en español; y, tercero, Saunders (1999) fue el único trabajo sobre la referencia en español como segunda lengua. No obstante, las muestras son orales y no escritas. Una breve reseña sobre estos trabajos aparece en Vargas-Víquez (2012, pp. 218-220).

\section{Algunas nociones relacionadas con la enseñanza de segundas lenguas}

El tema de la investigación obligó a establecer el concepto de “adquisición”, para lo cual es necesario aclarar también otras nociones propias del campo de la enseñanza de segundas lenguas como son "interlengua" y "error". 
En cuanto a “adquisición”, Ellis (1995, p. 14) explica que no se ha llegado a una distinción definitiva entre "adquisición” y "aprendizaje”; tampoco se ha podido determinar cuáles son las mejores evidencias de adquisición de un ítem en segundas lenguas: si como "emergencia", "establecimiento" o como "uso correcto". En otras palabras, el concepto puede entenderse al menos de tres maneras distintas y, por eso, fue indispensable determinar cuál de los tres significados dirigiría este análisis.

Por otro lado, y en estrecha relación con el concepto anterior, aparece el de "interlengua" que Selinker (1994, p. 231) considera como un sistema en sí mismo dentro del cual se dan los intentos de los aprendices para poner en práctica alguna norma de la lengua meta, tanto aciertos como desarciertos. Los desaciertos pueden ser considerados “errores”. En ese sentido, Ellis (1995, p. 19) plantea que los errores que provienen de la imitación del uso de la primera lengua (L1) del aprendiz son indicadores de transferencia y los errores similares a los del niño que adquiere esa lengua como L1 serían muestra de lenguaje creativo. Más concretamente, en cuanto al tema de este estudio, Saunders (1999, p. 14) señala que el uso de los recursos referenciales dentro de la interlengua puede ser visto como "no cohesivo" por parte de los hablantes nativos (HN).

Con estas nociones teóricas en mente, Alvarado-Gutiérrez y Vargas-Víquez (2009, p. 26) construyeron una definición operativa donde se entiende por adquisición "el mayor nivel de desarrollo de la interlengua alcanzado por un aprendiz o la producción de una estructura sin errores o con un nivel muy bajo de error, casi igual que un hablante nativo (HN)". De aquí nace la necesidad de analizar un grupo de HN del español. Valga aclarar que la producción nativa no fue el foco del estudio; sin embargo, la información obtenida a partir de este corpus serviría como parámetro de control de los datos de los hablantes no nativos (HNN). 


\section{La referencia}

La “cohesión" es uno de los rasgos más relevantes y visibles de la coherencia textual. Los conectores, la puntuación y los elementos referenciales son las formas cohesivas más importantes y son llamados por Halliday y Hasan (1976, p. 37) “ítems referenciales”, pues tienen la propiedad de relacionar un elemento con otro a lo largo del texto para establecer su identidad.

Como lo muestra la Figura 1, según la localización del elemento a que remita el mecanismo, la referencia puede ser de dos tipos: la referencia exofórica es la identificación extratextual y la referencia endofórica es intratextual (Halliday y Hasan, 1976). Por ejemplo, en me gustan aquellas al lado de la mesa, el pronombre "aquellas" refiere a un elemento extratextual que aquí tendríamos que imaginar (lámparas, cortinas, sillas,...) y, por lo tanto, es exofórico. En cambio, en traiga los libros y ordénelos en estos estantes, el pronombre "los" refiere a "los libros" que ya ha sido mencionada dentro del texto, esto es una referencia endofórica. La endófora puede remitir hacia atrás (anáfora) o hacia adelante (catáfora) en el texto, ambas son cohesivas; no así la exófora, pues no establece relaciones con elementos internos del texto, sino externos a él.



Figura 1. La referencia

Fuente: tomado de Halliday y Hasan (1976, p. 33) 
Halliday y Hasan (1976, p. 3) también señalan que cuando aparece una secuencia de formas que remiten a un mismo participante, se forma una cadena correferencial; como en Marta vino,

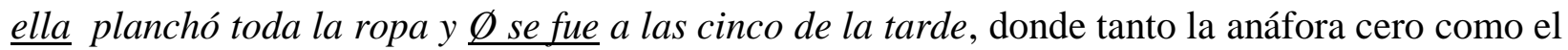
pronombre refieren al mismo participante: Marta $\leftarrow$ ella $\leftarrow \varnothing$ se fue. Precisamente, estas secuencias correferenciales fueron buscadas, extraídas y sistematizadas en la investigación de Alvarado-Gutiérrez y Vargas-Víquez (2009) como principales datos para el análisis.

Por otro lado, según el tipo de información que proporciona, la referencia puede ser: de persona, de ubicación demostrativa y de comparación. La categoría de persona es la que codifica la referencia personal, la localización demostrativa se hace según sea la proximidad del referente y las relaciones de igualdad, desigualdad o semejanza son las que funcionan en la comparativa (Halliday y Hasan, 1976, pp. 37-39).

\subsection{La referencia personal}

Todo texto introduce participantes que deben ser claramente identificados por el receptor textual; la deficiencia en dicha identificación produce escritos oscuros, difíciles de entender y que pueden ser malinterpretados. Es por esta razón que el escritor debe asegurarse de que su lector tenga las marcas lingüísticas necesarias para saber de quién o de qué se está hablando en el desarrollo del discurso. Los actores o participantes del texto se codifican por medio de lo que Halliday y Hasan (1976, p. 43) llaman "sistema de persona". Estos autores aplican ese nombre porque son varias las formas gramaticales que poseen la propiedad de mantener la referencia personal: los pronombres personales (tanto los de sujeto como los de objeto), los determinantes posesivos y los pronombres posesivos. La Tabla 1 contiene las formas de referencia personal para el español. 


\section{Tabla 1}

\section{Versión para el español de Referencia personal}

\section{ROLES DE HABLA OTROS ROLES}

\begin{tabular}{ll}
\hline EMISOR & RECEPTOR \\
\hline Yo, me, mí (a) (s) & Tú, te, tu, tuyo (a) Él, ella, le, su, suyo
\end{tabular}

\begin{tabular}{lll}
\hline Yo, me, mío (a) (s) & $\begin{array}{l}\text { Tú, te, tu, tuyo (a) } \\
\text { (s) }\end{array}$ & $\begin{array}{l}\text { Él, ella, le, su, suyo } \\
\text { (a) (s) }\end{array}$ \\
& $\begin{array}{ll}\text { Usted, le, su, suyo } \\
\text { (a) (s), vos }\end{array}$ \\
\hline $\begin{array}{l}\text { Nosotros, nos, } \\
\text { nuestro (a) (s) }\end{array}$ & Ustedes, les, su, & Ellos, ellas, les, \\
& suyo (a) (s) & sus, suyo (a)(s) \\
\hline Exofóricos & Exofóricos & Endofóricos \\
& & Anafóricos \\
\multicolumn{2}{c}{ Fuente: tomado de Alvarado-Gutiérrez y Vargas-Víquez (2009). }
\end{tabular}

Respecto a esta categoría, hay que tener en cuenta dos aspectos particulares. Por un lado, los lazos referenciales de tercera persona llenan su significado con información de participantes que han sido mencionados antes directamente en el texto, son endofóricos; mientras que los participantes que completan el sentido de los de primera y segunda persona pertenecen al contexto de la situación comunicativa, son exofóricos; por eso Halliday y Hasan (1976) consideran cohesivos solo los mecanismos de tercera persona. Esta es la razón por la cual, en el análisis de las muestras del estudio, se incluyen solo las realizaciones que corresponden a la tercera persona singular. Por otro lado, los pronombres personales se usan si se dan ciertas condiciones discursivas particulares; según Sánchez-Avendaño (2006, p. 227), “aparecen en situaciones específicas donde el productor textual considera que la forma referencial está activa y resulta accesible o fácilmente recuperable para su interlocutor". 


\subsection{Referencia demostrativa}

Dentro de la referencia demostrativa se encuentran los modificadores determinantes del grupo nominal, tanto los demostrativos (este, esta, estos, estas, ese, esa, esos, esas, aquel, aquella, aquellos, aquellas) como los artículos. Estos últimos tienen diferentes funciones dentro del discurso. Así, los artículos definidos señalan que el participante es identificable, ya sea porque es una entidad única e inconfundible o porque ya se había introducido antes en el texto. En cambio, los artículos indefinidos, generalmente, se usan cuando el referente se menciona por primera vez (Sánchez-Avendaño, 2006, p. 274).

\subsection{La anáfora cero}

A este mecanismo se le conoce en la gramática tradicional como sujeto tácito. Más recientemente se le llama también elipsis del sujeto y sujeto nulo. Se trata de la forma más frecuente de referir en español y se realiza mediante la omisión explícita del sujeto una vez que ha aparecido por primera vez y hasta que se introduce un nuevo sujeto. Esto es posible porque, en español, el sujeto se marca morfológicamente en el verbo, lo que no ocurre con ningún otro argumento. En cuanto a la alternancia de este recurso con otras formas referenciales, esta depende más bien de las posibilidades de identificación que tenga la entidad durante el desarrollo del texto:

La anáfora cero aparece cuando el productor textual considera que la forma referencial está activa y resulta accesible o fácilmente recuperable para su interlocutor. Pero cuando el hablante considera que la identificación del referente no puede realizarse de forma expedita por medio de un pronombre/anáfora cero, codifica nuevamente la entidad por medio de un GN (Sánchez-Avendaño, 2006, p. 277). 
La investigación de Alvarado-Gutiérrez y Vargas-Víquez (2009) se concentró en la referencia personal, en la referencia demostrativa: el artículo definido e indefinido y en la anáfora cero. Se extrajeron las realizaciones de siete diferentes categorías o clases gramaticales: los núcleos, que incluyen el sustantivo común, un nombre propio o un pronombre; los determinantes, que agrupan los posesivos y los artículos definidos e indefinidos; y, además, la anáfora cero.

\section{Metodología}

Alvarado-Gutiérrez y Vargas-Víquez (2009) analizaron un corpus de sesenta muestras escritas por hablantes no nativos del español en los diferentes niveles que establece ACTFL (2001). Además, aplicaron el mismo análisis en seis muestras de hablantes nativos, con el fin de que sirviera de grupo de control. En este artículo, se ofrecen los resultados que obtuvieron las investigadoras al comparar los datos de los tres niveles con las muestras nativas.

Tanto los informantes nativos como los no nativos describieron por escrito a los miembros de su familia. La transcripción respeta la puntuación y la ortografía que usaron los productores textuales. Además, cada redacción se tituló con iniciales mayúsculas según el corpus al que perteneciera, es decir, HN: Hablante Nativo y HNN: Hablante no nativo y también con un número identificador. A las muestras de HNN se les añadió otra letra que indica su nivel de desempeño lingüístico, según ACTFL (2001): A (Avanzado); I (Intermedio); P (Principiante). Asimismo, cada texto se segmentó en líneas que corresponden al uso de punto por parte del informante. Por ejemplo, HN 1, L4 es la muestra número uno del corpus de hablantes nativos en la línea 4. 


\section{Hablante Nativo 1 \\ Cadenas referenciales:}

(Alvarado-Gutiérrez y Vargas Víquez, 2009)

1 Mi familia está compuesta por cinco miembros: mis padres, mis dos hermanos y yo.

2 Mi papá1 se llama Wálter y desde muy pequeño ha trabajado 1 muy duro para poder darnos las necesidades básicas y nuestra profesión, lo cual representa para él ${ }_{1}$ lo fundamental para que, tanto mis hermanos como yo, podamos "defendernos" en la carrera de la vida.

3 Toda su $_{1}$ vida trabajó 1 en el área de venta de repuestos, y hace ya como seis o siete años que $\mathrm{se}_{1}$ pensionó 1 .

4 Tiene $_{1}$ muchísimas virtudes, sin embargo las que más le ${ }_{1}$ admiro son su sabio $_{1}$ consejo ( siempre tiene $_{1}$ una palabra adecuada cuando uno más lo necesita) y su $\mathrm{su}_{1}$ paciencia.

5 Mi mamá2 se llama Marielos, y es 2 ama de casa, por lo que se 2 dedicó 2 por completo a las labores de la casa - que muchas veces son mucho más agotantes de lo que cualquiera se imagina - y por supuesto a la crianza de mis hermanos y yo. Siempre se preocupaba 2 por nuestras labores y por sobre todo el estudio de cada uno de nosotros, ya que, como ella 2 suele decir: “....el estudio es la mejor herencia que se le puede dar a un hijo, por lo tanto tienen que aprovecharlo...".

6 Actualmente, ella 2 explota muy bien su sentido de servicio a los demás, por lo que participa $_{2}$ en varios grupos de la Iglesia Católica que brindan ayuda social a los más necesitados y a grupos de la tercera edad.

7 De ella 2 siempre he admirado $\mathrm{su}_{2}$ entrega desinteresada y $\mathrm{su}_{2}$ excelente capacidad para planificar las cosas, pensando siempre en el futuro...

8 Ah!, se me olvidaba..., también cocina 2 riquísimo!...tiene 2 una excelente "cuchara".

9 Mi hermano 3 mayor se llama Wálter, y es 3 Administrador de Empresas.

10 Sin embargo, ha realizado 3 estudios para "corredor de bolsa", por lo que actualmente labora 3 en esta área en el Banco HSBC.

11 Es$_{3}$ casado y tiene 3 tres hijos preciosos. 
$12 \mathrm{Le}_{3}$ encanta la música, por lo que pasa 3 bastante rato de $\mathrm{su}_{3}$ tiempo libre haciendo mezclas por computadora de la música que más le 3 gusta.

13 Indudablemente, una de las cualidades que me impresionan y enorgullecen de él 3 es $\mathrm{su}_{3}$ enorme nobleza.

14 Y por último, pero no menos importante, mi hermana 4 Vanessa, quien es 4 Farmacéutica.

15 Ella 4 ha sido la más esforzada de los tres en cuanto al estudio se refiere; siempre fue $_{4}$ la más aplicada y la que dedicaba más tiempo a estudiar para los exámenes.

16 Actualmente labora 4 como visitadora médica para la empresa Novartis.

17 Siempre ha tenido 4 muy buenas aptitudes para el dibujo, y en ocasiones ha llevado $_{4}$ cursos libres de pintura.

18 Es4 casada y tiene 4 aproximadamente 6 meses de embarazo (si no me equivoco), noticia que toda la familia recibió con muchísima alegría e ilusión, ya que al ser la única mujer de los hermanos, todos - en el fondo de nuestro corazón - soñábamos con chinear un peque de mi hermanita4.

$19 \mathrm{~A}$ ella $4 \mathrm{le}_{4}$ admiro su 4 perseverancia para conseguir todas sus 4 metas, y su manera tan dulce y especial para tratar a los demás.

Puede observarse en esta muestra que a las secuencias de cada pariente del productor textual se les colocó un mismo subíndice. En la línea 5, se introduce a "la mamá2" con subíndice 2; esta marca aparece cada vez que un mecanismo referencial remite a este participante. Valga aclarar que el subíndice 0 se usó para marcar los participantes en tercera persona singular que no formaron cadenas referenciales porque solo aparecieron una vez en la redacción y que, por lo tanto, no se contabilizaron en esta investigación.

La información se sistematizó en una tabla de clasificación como la que aparece más adelante, donde la primera columna registra la línea en que aparece el mecanismo; la segunda columna registra la forma gramatical exacta; la tercera clasifica el mecanismo según su categoría 
gramatical; en la cuarta, se anota el nombre del referente según el parentesco donde empieza cada cadena referencial.

A continuación, se presenta la lista de las siglas que se usaron en esas tablas:

$\mathrm{SC}=$ sustantivo común,

$\mathrm{NP}=$ nombre propio,

$\mathrm{P}=$ pronombre,

$\mathrm{A}=$ anáfora cero,

DPos $=$ determinante posesivo,

SC-DIndef = sustantivo común con determinante indefinido,

SC-DDef = sustantivo común con determinante definido.

Tabla 2

Clasificación de las cadenas referenciales HN 1 


\begin{tabular}{|c|c|c|c|}
\hline Línea & Realización & Clase & Referente \\
\hline 2 & papá 1 & $\mathrm{SC}$ & El papá \\
\hline 2 & ha trabajado 1 & A & \\
\hline 2 & él ${ }_{1}$ & $\mathrm{P}$ & \\
\hline 3 & $\mathrm{su}_{1}$ & DPos & \\
\hline 3 & trabajó $_{1}$ & $\mathrm{~A}$ & \\
\hline 3 & $\mathrm{se}_{1}$ & $\mathrm{P}$ & \\
\hline 3 & pensionó $_{1}$ & A & \\
\hline 4 & tiene $_{1}$ & A & \\
\hline 4 & $\mathrm{le}_{1}$ & $\mathrm{P}$ & \\
\hline 4 & $\mathrm{su}_{1}$ & DPos & \\
\hline 4 & tiene $_{1}$ & A & \\
\hline 4 & $\mathrm{su}_{1}$ & DPos & \\
\hline 5 & mamá $_{2}$ & $\mathrm{SC}$ & La mamá \\
\hline 5 & $\mathrm{es}_{2}$ & $\mathrm{~A}$ & \\
\hline 5 & $\mathrm{se}_{2}$ & $\mathrm{P}$ & \\
\hline 5 & dedicó $_{2}$ & A & \\
\hline 5 & $\mathrm{Se}_{2}$ & $\mathrm{P}$ & \\
\hline 5 & se preocupaba 2 & A & \\
\hline 5 & ella $_{2}$ & $\mathrm{P}$ & \\
\hline 6 & ella $_{2}$ & $\mathrm{P}$ & \\
\hline 6 & $\mathrm{su}_{2}$ & DPos & \\
\hline 6 & participa $_{2}$ & A & \\
\hline 7 & ella $_{2}$ & $\mathrm{P}$ & \\
\hline 7 & $\mathrm{su}_{2}$ & DPos & \\
\hline 7 & $\mathrm{su}_{2}$ & DPos & \\
\hline 8 & $\operatorname{cocin}_{2}$ & $\mathrm{~A}$ & \\
\hline 8 & tiene $_{2}$ & A & \\
\hline 9 & hermano 3 & $\mathrm{SC}$ & El hermano \\
\hline 9 & $\mathrm{es}_{3}$ & $\mathrm{~A}$ & \\
\hline 10 & ha realizado 3 & A & \\
\hline 10 & labora $_{3}$ & A & \\
\hline 11 & $\mathrm{es}_{3}$ & A & \\
\hline 11 & tiene $_{3}$ & $\mathrm{~A}$ & \\
\hline 12 & $\mathrm{Le}_{3}$ & $\mathrm{P}$ & \\
\hline 12 & $\varnothing$ pasa $_{3}$ & A & \\
\hline
\end{tabular}




\begin{tabular}{ccc}
\hline 12 & $\mathrm{su}_{3}$ & DPos \\
\hline 12 & $\mathrm{le}_{3}$ & $\mathrm{P}$ \\
\hline 13 & $\mathrm{é}_{3}$ & $\mathrm{P}$ \\
\hline 13 & $\mathrm{su}_{3}$ & DPos \\
\hline
\end{tabular}

\begin{tabular}{ccc}
\hline 14 & hermana $_{4}$ & SC \\
\hline 14 & $\varnothing$ es4 & A \\
\hline 15 & Ella $_{4}$ & $\mathrm{P}$ \\
\hline 15 & $\varnothing$ fue $_{4}$ & $\mathrm{~A}$ \\
\hline 15 & la & $\mathrm{P}$ \\
\hline 16 & $\varnothing$ labora $_{4}$ & $\mathrm{~A}$ \\
\hline 17 & $\varnothing$ ha tenido 4 & $\mathrm{~A}$ \\
\hline 17 & $\varnothing$ ha llevado 4 & $\mathrm{~A}$ \\
\hline 18 & $\varnothing$ Es$_{4}$ & $\mathrm{~A}$ \\
\hline 18 & $\varnothing$ tiene $_{4}$ & $\mathrm{~A}$ \\
\hline 18 & hermanita $_{4}$ & $\mathrm{SC}$ \\
\hline 19 & ella $_{4}$ & $\mathrm{P}$ \\
\hline 19 & le $_{4}$ & $\mathrm{P}$ \\
\hline 19 & su $_{4}$ & DPos \\
\hline 19 & sus $_{4}$ & DPos \\
\hline 19 & su $_{4}$ & Dpos
\end{tabular}

En la investigación, a cada uno de esos cuadros se le agregó otro que resume la información y a la que se le llamó "Frecuencia de uso de las realizaciones según clase". Este mismo procedimiento que se acaba de describir se aplicó a todas las muestras, tanto del corpus nativo como del corpus no nativo. 


\section{Tabla 3}

Frecuencia de uso de las realizaciones según clase

\begin{tabular}{cc}
\hline SC & 5 \\
\hline NP & 0 \\
\hline P & 15 \\
\hline A & 24 \\
\hline DPos & 11 \\
\hline SC-DIndef. & 0 \\
\hline SC-DDef. & 0 \\
\hline Total de realizaciones en las cadenas analizadas & 55
\end{tabular}

\section{Resultados de las muestras de los hablantes nativos}

Como ya ha sido mencionado, para tener un parámetro en cuanto al uso nativo de los recursos de referencia se recopilaron seis redacciones escritas por adultos costarricenses. A estos informantes se les asignó la misma tarea que a los no nativos, esto es, describir a su familia nuclear (padres y hermanos). De esta manera, se identificaron las formas más usadas por el grupo de control y con estos datos se estableció el patrón de uso nativo que representa el mayor grado de adquisición de estos mecanismos. En la siguiente tabla se contabilizan los recursos según su clase gramatical:

\section{Tabla 4}


Frecuencia de uso de las realizaciones según su clase.

Hablantes nativos. Total de realizaciones: 287

\begin{tabular}{ccccccccc}
$\begin{array}{c}\text { Muestra/ } \\
\text { Clase }\end{array}$ & 1 & 2 & 3 & 4 & 5 & 6 & Total & $\%$ \\
\hline $\mathrm{A}$ & 24 & 13 & 18 & 31 & 21 & 9 & 116 & 40.41 \\
\hline $\mathrm{SC}$ & 5 & 7 & 6 & 7 & 18 & 14 & 57 & 19.86 \\
\hline $\mathrm{P}$ & 15 & 6 & 8 & 9 & 14 & 11 & 63 & 21.95 \\
\hline DPos & 11 & 3 & 0 & 8 & 7 & 9 & 38 & 13.24 \\
\hline NP & 0 & 0 & 0 & 6 & 1 & 0 & 7 & 2.43 \\
\hline SC-DIndef & 0 & 1 & 0 & 0 & 0 & 2 & 3 & 1.04 \\
\hline SC-DDef & 0 & 0 & 0 & 2 & 0 & 1 & 3 & 1.04 \\
\hline Total & $\mathbf{5 5}$ & $\mathbf{3 0}$ & $\mathbf{3 2}$ & $\mathbf{6 3}$ & $\mathbf{6 1}$ & $\mathbf{4 6}$ & $\mathbf{2 8 7}$ & $\mathbf{1 0 0}$ \\
\hline
\end{tabular}

datos indican que la anáfora cero es la forma más usada por los hablantes nativos, seguida del pronombre y luego del sustantivo común. El mecanismo que menos se encontró en el corpus nativo fue el sustantivo común con determinante definido. La Figura 2 ilustra el patrón de aparición de los recursos

Fuente: tomado de Alvarado-Gutiérrez y Vargas-Víquez (2009).

Los datos indican que la anáfora cero es la forma más usada por los hablantes nativos, seguida del pronombre y luego del sustantivo común. El mecanismo que menos se encontró en el corpus nativo fue el sustantivo común con determinante definido. La Figura 2 ilustra el patrón de aparición de los recursos referenciales en las muestras de los HN.

A $>$ P $>$ SC $>$ DPos $>$ NP $>$ SC-DIndef $>$ SC-Ddef

Figura 2. Patrón de uso de los mecanismos referenciales HN

Fuente: tomado de Alvarado-Gutiérrez y Vargas-Víquez (2009).

\section{Comparación de los tres niveles de hablantes no nativos}


Al iniciar el estudio, se había planteado la hipótesis de que la frecuencia de uso de estos mecanismos tendería a parecerse cada vez más a la de los hablantes nativos conforme se avanzara en los niveles. No obstante, tal y como se aprecia en la Tabla 5, se encontró el mismo orden o patrón en todos los niveles. Esto es, el mecanismo más usado por los estudiantes fue siempre el pronombre; luego, el sustantivo común; en tercer lugar, la anáfora cero; y, en cuarto puesto, los determinantes posesivos de tercera persona singular.

Tabla 5. Frecuencia de uso de las realizaciones según su clase en cada nivel. Total de realizaciones

\begin{tabular}{lllllllll}
$\begin{array}{l}\text { Clase/ } \\
\text { Nivel }\end{array}$ & $\mathbf{P}$ & SC & A & DPos & NP & DIndef & DDef & Total \\
\hline $\begin{array}{l}\text { Nivel } \\
\text { P }\end{array}$ & 171 & 122 & 40 & 31 & 26 & 10 & 0 & 400 \\
\hline Nivel I & 349 & 180 & 95 & 53 & 28 & 15 & 1 & 721 \\
\hline $\begin{array}{l}\text { Nivel } \\
\text { A }\end{array}$ & 210 & 162 & 112 & 64 & 39 & 21 & 7 & 615 \\
\hline Total & 730 & 464 & 247 & 148 & 93 & 46 & 8 & 1736
\end{tabular}

Fuente: tomado de Alvarado-Gutiérrez y Vargas-Víquez (2009).

Las barras de la Figura 3 ilustran cómo el sustantivo común varía muy poco la frecuencia de uso en los tres niveles; el nombre propio y los pronombres se usan más en el nivel intermedio. Por su parte, la anáfora cero y los determinantes posesivos de tercera persona van ascendiendo según avanza el nivel; sin embargo, las diferencias en la cantidad de realizaciones usadas no son tan significativas como para provocar un quiebre en el patrón de uso. Los otros mecanismos 
(sustantivo común con determinante definido y sustantivo común con determinante indefinido) representan porcentajes muy pequeños.

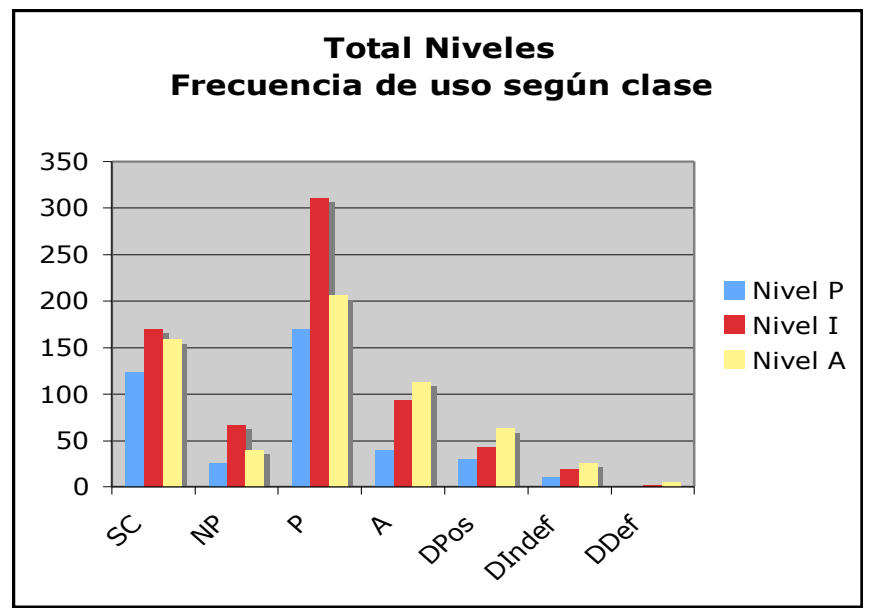

Figura 3. Frecuencia de uso de las realizaciones según su clase en cada nivel Fuente: tomado de Alvarado-Gutiérrez y Vargas-Víquez (2009).

De esta manera, el orden de los mecanismos de referencia según la frecuencia de uso por parte de los hablantes no nativos, en los tres niveles de dominio lingüístico, puede representarse de la siguiente forma:

$\mathrm{P}>\mathrm{SC}>\mathrm{A}>\mathrm{DPos}>\mathrm{NP}>\mathrm{SC}$-DIndef $>$ SC-DDef

Figura 4. Patrón de uso de los mecanismos referenciales. HNN Fuente: tomado de Alvarado-Gutiérrez y Vargas-Víquez (2009)

7. Comparación de los resultados de los tres niveles con los de los hablantes nativos 
A partir de la pregunta orientadora del trabajo de si existe un patrón de adquisición de los mecanismos gramaticales de referencia en estudiantes de español como segunda lengua y luego del análisis de los datos, se puede decir que existe un patrón de uso de los HNN que es el mismo en los tres niveles; sin embargo, dicho patrón no es de "adquisición" de las formas referenciales, pues no se acerca al patrón nativo. Además, al comparar los resultados de los HNN y de los HN en el Cuadro 6, se nota que la principal diferencia es que el hablante nativo usa en primer lugar la anáfora cero $(40.41 \%)$, con un amplio margen de diferencia entre este mecanismo y su más cercano seguidor, que es el sustantivo común (19.86 \%); en cambio, los aprendices usan el pronombre en primerísimo lugar $(41.76 \%)$ y también con un amplio margen de diferencia con el sustantivo común $(27.93 \%)$, que es la forma que le sigue. La anáfora cero, prevalente en la frecuencia nativa, ocupa en la frecuencia no nativa un tercer lugar.

Tabla 6. Comparación de uso de cada tipo de realización según clase HN y HNN

\begin{tabular}{lll} 
Clase & Porcentaje HN & Porcentaje HNN \\
\hline $\mathrm{A}$ & 40.41 & 13.79 \\
\hline $\mathrm{SC}$ & 19.86 & 27.93 \\
\hline $\mathrm{P}$ & 21.95 & 41.76 \\
\hline DPos & 13.24 & 8.5 \\
\hline $\mathrm{NP}$ & 2.43 & 5.57 \\
\hline SC-DIndef & 1.04 & 2.66 \\
\hline SC-DDef & 1.04 & 0.42 \\
\hline Total & $100 \%$ & $100 \%$
\end{tabular}

Fuente: tomado de Alvarado-Gutiérrez y Vargas-Víquez (2009). 
En la Tabla 7 aparecen los porcentajes de uso de cada mecanismo tanto en el corpus de HN como en los tres niveles de HNN. Obsérvese que en ninguno de los niveles de HNN se usan los mecanismos en estudio como lo muestran los datos de los HN.

Tabla 7. Comparación de uso de cada tipo de realización según su clase entre HN y cada uno de los niveles

\begin{tabular}{lllll} 
Clase & HN & Principiante & Intermedio & Avanzado \\
\hline $\mathrm{A}$ & 40.41 & 10 & 13.17 & 18.21 \\
\hline $\mathrm{SC}$ & 19.86 & 30.5 & 24.96 & 26.34 \\
\hline $\mathrm{P}$ & 21.95 & 42.75 & 48.40 & 34.14 \\
\hline DPos & 13.24 & 7.75 & 7.35 & 10.40 \\
\hline NP & 2.43 & 6.5 & 3.88 & 6.34 \\
\hline SC-DIndef & 1.04 & 2.5 & 2.08 & 3.41 \\
\hline SC-DDef & 1.04 & 0 & 0.13 & 1.13 \\
\hline Total & $100 \%$ & $100 \%$ & $100 \%$ & $100 \%$
\end{tabular}

Fuente: tomado de Alvarado-Gutiérrez y Vargas-Víquez (2009).

Para verlo de una forma más gráfica, se ofrece la Figura 5, donde se aprecia la diferencia entre ambos patrones. 


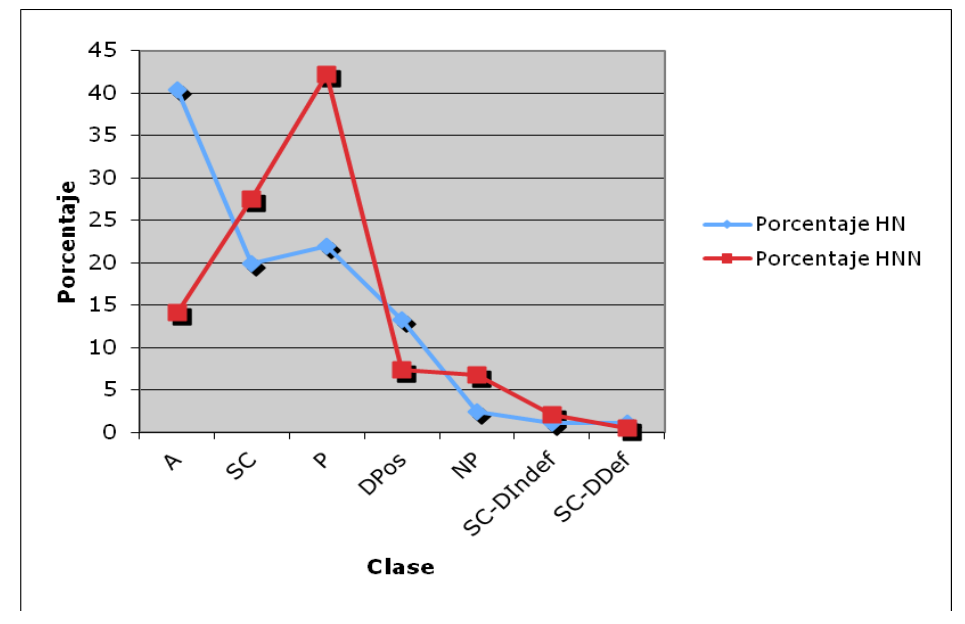

\section{Figura 5. Comparación entre del uso de HN y de HNN de cada tipo de realización según su clase}

Fuente: tomado de Alvarado-Gutiérrez y Vargas-Víquez (2009).

Nótese en la figura anterior el uso proporcionalmente inverso de la anáfora cero y el pronombre en ambos grupos de informantes, lo cual puede tener relación con el hecho que plantea Saunders (1999, p. 1) de que "el español permite tanto el sujeto nulo como el explícito; mientras que el inglés requiere el sujeto explícito”. Por consiguiente, puede tratarse de transferencia de la L1 a la L2, en este caso del inglés al español; los informantes usan los pronombres en español para mantener activo un referente como lo harían en inglés, y, como se había mencionado antes, según Saunders (1999, p. 14) este uso no nativo es percibido como "no cohesivo" por parte de los HN. Alvarado-Gutiérrez y Vargas-Víquez (2009) reconocen el valor de la transferencia durante las diferentes etapas de la interlengua; no obstante, llama la atención que, aún en las muestras de nivel avanzado, el uso de la anáfora cero se aleja mucho del habla nativa. Es decir, la transferencia puede considerarse una estrategia natural de aprendizaje, pero sería esperable que el aprendiz se desprenda de los modelos de su L1 conforme tiene mayor contacto con la L2.

Por otro lado, se pueden establecer varias condiciones que rigen la elección de un determinado mecanismo referencial. Sánchez-Avendaño (2006, p. 283) señala que "el hablante 
elige el grupo nominal pleno con determinante indefinido para introducir nuevos participantes en el texto; sin embargo, una vez que se considera que el participante es identificable, la referencia se mantendrá por medio de pronombres o de anáfora cero”. Asimismo, Saunders (1999, p. 4) menciona que el sujeto nulo deberá usarse en español a menos que haya una razón para usar el sujeto explícito. Sus investigaciones han identificado dos circunstancias para que esto ocurra: primero, para desambiguar un referente; y segundo, para enfatizar a un participante. El corpus de los HN calza con estos planteamientos en cuanto a la preferencia por la anáfora cero que solo se modifica en casos muy concretos y para asegurar la identificación o dar énfasis a un participante. Sánchez-Avendaño (2006, p. 283) también señala que el grupo nominal con determinante indefinido es el mecanismo más frecuente a la hora de introducir un participante en el texto; no obstante, esto no aparece en ninguno de los dos corpus de la investigación. La explicación para esta particularidad radica en el tema de la redacción (la familia), y que provoca que, en la mayoría de los casos, el mecanismo de introducción de los participantes sea el posesivo: mi papá, mi mamá, mi hermano.

\section{Conclusiones}

La principal conclusión a la que llegó esta investigación fue que los hablantes no nativos no adquirieron los mecanismos de referencia en ninguno de los tres niveles de competencia lingüística. Hay que recordar que se entiende adquisición como nivel de interlengua más desarrollado que se acerca mucho a la producción nativa (Alvarado-Gutiérrez y Vargas-Víquez, 2009). Planteado con otras palabras, los HNN logran la tarea comunicativa de describir a sus familias y lo hacen con las estrategias que su nivel de interlengua les provee que son diferentes a las estrategias que usan los HN (Saunders, 1999, p. 14); por eso y para ser consecuentes con la 
definición operativa de "adquisición", se concluye que no han adquirido los mecanismos referenciales. No obstante, sí se encontró un patrón (no de adquisición), sino de uso dentro de la interlengua, el cual se grafica de la siguiente forma:

$$
\text { P }>\text { SC }>\text { A }>\text { DPos }>\text { NP }>\text { SC-DIndef }>\text { SC-DDef }
$$

\section{Figura 6. Patrón de uso de los mecanismos referenciales. HNN}

Fuente: tomado de Alvarado-Gutiérrez y Vargas-Víquez (2009).

Una de las razones que pueden explicar la diferencia de los patrones de uso es la transferencia, pues los errores de las muestras no nativas reflejan las estrategias de su lengua materna (el inglés) y no las del español. Por ser el español una lengua de sujeto nulo, la forma apropiada de mantener la referencia en esta lengua es por medio de la anáfora cero (a la cual llama pronombre nulo). Sin embargo, el hablante nativo recurrirá a otros mecanismos de mantenimiento de la referencia bajo dos condiciones: cuando sea necesario desambiguar un referente, cuando se quiera dar énfasis a un participante (Saunders, 1999, p. 4).

Otras conclusiones importantes son las siguientes:

a) El patrón de adquisición de los mecanismos de referencia por estudiantes de español como segunda lengua no varía de un nivel a otro durante un año de contacto con la lengua, lo cual despierta dos preguntas: por un lado, ¿qué ocurriría si un estudio similar se hiciera con informantes con más tiempo de contacto con el español? Y, por otro, ¿en qué momento empiezan a acercarse al uso nativo?

b) En el aula y ante este panorama, el profesor de español como segunda lengua podría acelerar la adquisición de estos recursos si incorpora en sus lecciones la enseñanza, no solo la clasificación 
gramatical de las formas referenciales aisladamente, sino también las condiciones discursivas que explican que su disposición en el texto no se da en forma arbitraria, tal como lo exponen SánchezAvendaño (2006) y Saunders (1999).

c) Además, es necesario producir materiales didácticos, pues el fenómeno cohesivo de la referencia no suele explicarse en los manuales de enseñanza de ESL; generalmente lo que se encuentran son cuadros taxonómicos de las formas lingüísticas según su categoría gramatical. Sería conveniente presentarlas más bien como una clase transversal de recursos que comparten su capacidad de referir, ya que los resultados indican que el mero conocimiento de las formas gramaticales de manera aislada no es suficiente para que el aprendiz se apropie de las funciones discursivas que cada una desempeña en el texto.

\section{Bibliografía}

Alvarado-Gutiérrez, Miriam y Vargas-Víquez, Silvia. (2009). Adquisición de los mecanismos de referencia por estudiantes de español como segunda lengua (Tesis de maestría). Universidad de Costa Rica.

American Council on the Teaching of Foreign Languages Inc. [ACTFL]. (2001). Criterios de Proficiencia-Escritura. Recuperado de http://www.actfl.org

Calsamiglia Blancafort, Helena y Tusón Valls, Amparo. (1999). Las cosas del decir. Manual de análisis del discurso. Barcelona: Ariel.

Chiang, Steve. (1999). Assessing grammatical and textual features in L2 writing samples. The case of French as a Foreing Language. The Modern Language Journal, 83, 219-232.

Ellis, Rod. (1985). Understanding Second Language Acquisition. Oxford: Oxford University Press. 
Govardhan, Anam K. (1994). A discourse analysis of ESL student writing (Tesis doctoral). Universidad del Norte de Illinois, Estados Unidos.

Halliday, M.A.K. y Ruqaiya, Hasan. (1976). Cohesion in English. Nueva York: Longman.

Lee, James F. (2003). Clitics. Cognitive and Linguistic Perspectives on the Acquisition of Object Pronouns in Spanish as a Second Language. En Lafford y Salaberry (Ed.), Spanisn Second Language Acquisition (98-128). USA: Georgetown University Press

Pongsiriwet, Charuporn. (2001). Relationships among grammatical accuracy, discourse features, and the quality of second language writing: The case of Thai EFL learners (Tesis doctoral). Universidad de Virginia del Oeste, Estados Unidos.

Reynolds, Dudley Walton. (1996). Repetition in second language writing (Tesis doctoral). Universidad de Indiana, Estados Unidos.

Robinson, Maisah Bint-Patrick. (1994). Composition teachers' criteria for good writing (Tesis doctoral). Universidad Estatal de Georgia, Estados.

Sánchez-Avendaño, Carlos. (2006). La forma del grupo nominal en español hablado. Un caso de gramática del discurso frente a gramática de la palabra. Revista de Filología y Lingüística, 32 (2), 261-289.

Saunders, Joy Kathleen. (1999). Null and overt references in Spanish second language acquisition: A discourse perspective (Tesis doctoral). Universidad de Texas en Austin, Estados Unidos.

Selinker, Larry. (1994). Rediscovering Interlanguage. New York: Longman Inc.

Shi, Huifen. (1993). The relation between freshman writing coherence and cohesion, functional roles, and cognitive strategies (Tesis doctoral). Universidad de Indiana en Pensilvania, Estados Unidos.

Teijeira-Rodríguez, María del Carmen, Van Esch, Kees, y de Haan, Pieter. (2005). La coherencia y la cohesión en textos escritos por estudiantes holandeses de español como LE. Estudios de Lingüística Aplicada, 23 (041), 67-100. Recuperado de http://redalyc.uaemex.m /redalyc/src/inicio/ArtPdfRed.jsp?Cve=58804105 
Tornqvist, Linnea. (2004). The use of coherence markers by Hispanic North American college writers in their essays in English and Spanish: Contrastive or comparative rhetoric? (Tesis doctoral). Universidad Estatal de Nueva Jersey, Estados Unidos.

Vargas-Víquez, Silvia. (2012). La adquisición de los recursos gramaticales de referencia por estudiantes de español como segunda lengua en el nivel principiante. Revista de Filología y Lingüística de la Universidad de Costa Rica, 38 (2), 199-214.

Vargas-Víquez, Silvia. (2014). Adquisición de los recursos gramaticales de referencia por estudiantes de español como segunda lengua en el nivel avanzado. Revista de Filología y Lingüística de la Universidad de Costa Rica, 40 (1), 217-238.

Vines, Joe Edwin. (1997). An assessment of the effects of computer-based writing instruction upon the teaching of English as a Second Language (Tesis doctoral). Universidad de Florida, Estados Unidos.

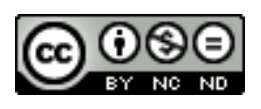

